\title{
Supplementary Information for a new OCO-2 cloud flagging and rapid retrieval of marine boundary layer cloud properties
}

\author{
Mark Richardson ${ }^{1,2}$, Matthew D. Lebsock ${ }^{1}$, James McDuffie ${ }^{1}$, Graeme L. Stephens ${ }^{1,3}$ \\ ${ }^{1}$ Jet Propulsion Laboratory, California Institute of Technology, Pasadena, CA 91109, USA \\ $5 \quad 2$ Joint Institute for Regional Earth System Science and Engineering, University of California, Los Angeles, CA 90095, USA \\ 3Department of Meteorology, University of Reading, RG6 7BE, UK
}

Correspondence to: Mark Richardson (markr@jpl.nasa.gov)

\section{Selection of classifier}

We considered 13 combinations of observables and 6 classifier setups, including $k$-nearest neighbours (KN with 12- or 25

10 neighbours), random forest (RF) and multi-layer perceptron (MLP with 1-, 2- or 3-layers), taking the default sklearn class properties unless specified otherwise. The observations are combinations of the continuum radiances or super-channel Aband ratios as described in Supplementary Table 1, which also provides the input dataset names and the selected classifier information.

Performance metrics are from the standard confusion matrix elements: true positive (TP), false positive (FP), false negative

15 (FN) and true negative (TN) as described in the main manuscript. The accuracy score is the trace of the confusion matrix, i.e. $\mathrm{TP}+\mathrm{TN}$, and we also inspect the following combinations:

(i) $\mathrm{FN} /(\mathrm{TP}+\mathrm{FN})$, i.e. the fraction of real cases that are missed,

(ii) $\mathrm{FP} /(\mathrm{TP}+\mathrm{FP})$, i.e. the fraction of classifier positives which are not true clouds,

(iii) $\quad \mathrm{FN} /(\mathrm{TN}+\mathrm{FN})$, i.e. the fraction of classifier negatives which are not truly negatives,

20 We wish to minimise (i) - (iii), with (i) representing the loss of potentially good retrievals, (ii) is related to wasted computational resources on footprints which are unlikely to be good retrievals and (iii) quantifies the fraction of negatives which are truly likely to be cloud free. These statistics are calculated for each classifier and observable combination for the independent (non-training) 250,000 footprint sample and are displayed in Supplementary Figure 1, which makes clear that the inclusion of the band ratios improves the accuracy score for all classifiers. Panels (b) - (d) show a range of performances

25 and we selected the 3 layer neural network with the 3chan_10thrat observations. This shows among the lowest rate of missed cases without a large spike in false positives, although there is no clear objectively best choice. 
(a)

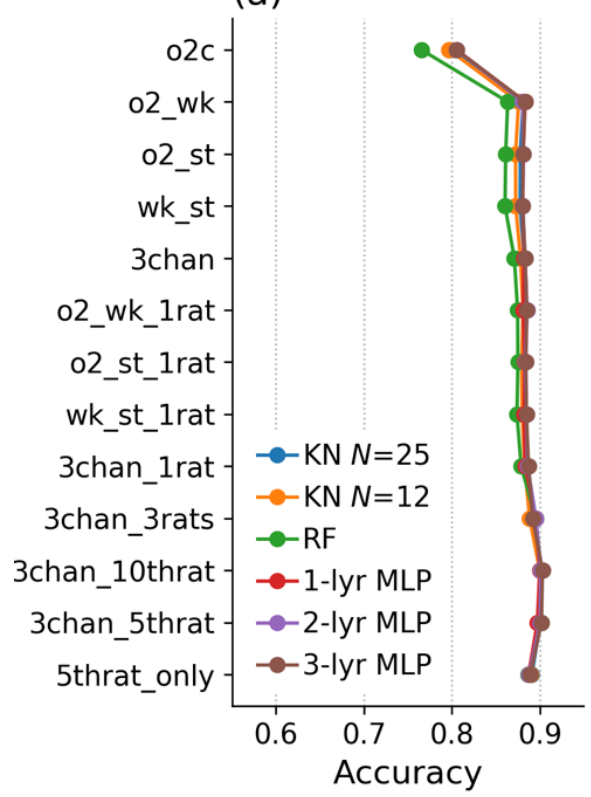

CALIPSO Liquid Cloud, MODIS $\tau>1$

(b)

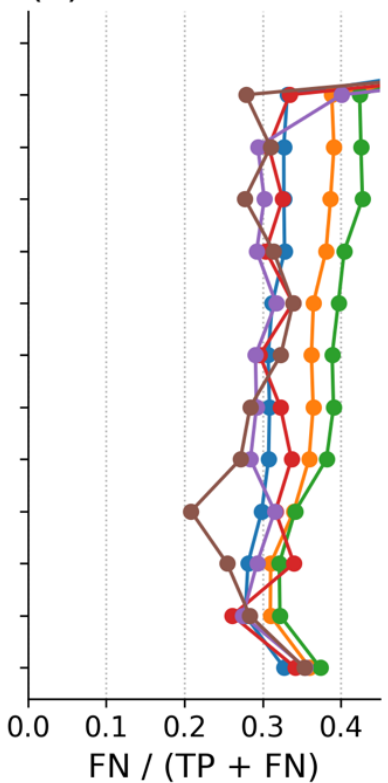

(c)

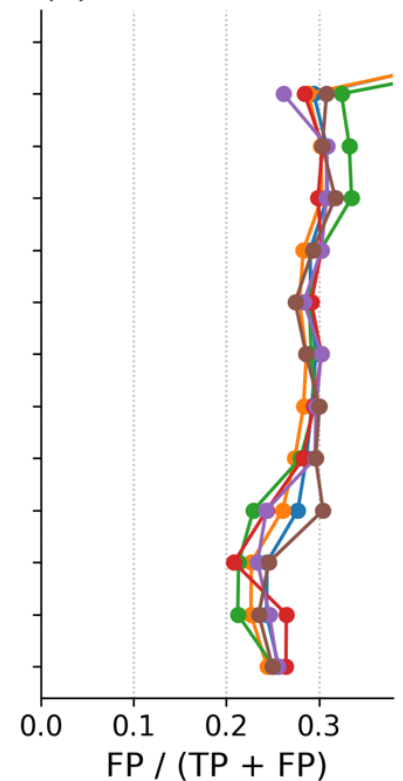

(d)

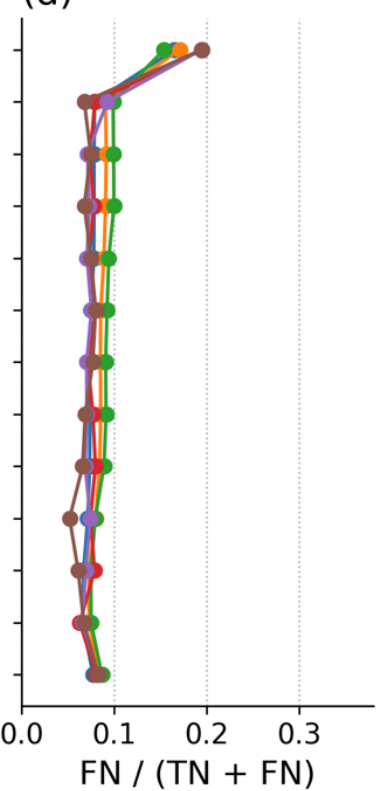

30 Supplementary Figure 1. Performance statistics of each tested classifier for each observable. Classifiers are labelled in legend, for $k$-nearest neighbours (KN, with $N$ neighbours), Random Forest $(R F)$ and multi-layer perceptron (MLP, with 1-, 2- or 3 hidden layers). Observations refer to $\mu_{0}^{-1} I$ continua for the $\mathrm{O}_{2}$ A-band (o2), weak $\mathrm{CO}_{2}$ band (wk) and strong $\mathrm{CO}_{2}$ band (st) while "rat" refers to the radiance ratio corrected for $\mu_{0}$ as described in Supplementary Table 1.

Supplementary Table 1. Properties used in the classifier along with sources

\begin{tabular}{|l|l|l|}
\hline Property & Value or short name & Data source or calculation method \\
\hline Hidden layer sizes & $(100,50,25)$ & hidden_layer_sizes in sklearn.neural_networks.MLPClassifier \\
\hline Cloud Classification flag & 1 & $\begin{array}{l}\text { CALIPSO 01 kmCLay Feature_Classification_Flag }=2 \text { (i.e. } \\
\text { liquid), MODIS MYD061KM Cloud_Optical_Thickness }>1, \\
\text { only one retrieved CALIPSO layer } \\
\text { CALIPSO non-liquid or not retrieved, OR MODIS } \tau<1 \text { or not } \\
\text { retrieved }\end{array}$ \\
& 0 & $\begin{array}{l}\text { SoundingGeometry/sounding_solar_zenith } \\
\text { RadianceMeasurements/rad_continuum_o2 } \\
\text { RadianceMeasurements/rad_continuum_wco2 } \\
\text { RadianceMeasurements/rad_continuum_sco2 } \\
\text { RadianceMeasurements/radiance_o2, ranked from brightest, } \\
\text { non-overlapping 10 channel mean, then every tenth index from } \\
\text { O2 continuum } I o 2, c \\
\text { Weak CO2 continuum } I_{w k, c} \\
\text { Strong CO2 continuum } I_{s t, c} \\
\text { A-band ratio }-\ln \left(\frac{I}{I_{c}}\right)\left(\mu_{0}+1\right)^{-1}\end{array}$ \\
& \begin{tabular}{l} 
SZA inclusive (first index $=1)$. \\
\hline
\end{tabular}
\end{tabular}




\section{Determination of surface albedo}

To estimate the equivalent Lambertian surface we used refractor's surface albedo estimator, which takes instrument information, the band continuum radiance and solar irradiance to provide a footprint surface albedo. To obtain this by SZA, we selected 1,000 random footprints in each $5^{\circ} \mathrm{SZA}$ bin from $20-45^{\circ}$ inclusive, provided that they were over ocean and that they were flagged as confidently clear by MODIS. The median refractor estimate of each of these 1,000 values was

40 used, and the values are in Supplementary Table 2. The albedos were interpolated from this table onto each LUT SZA, with the $\mathrm{SZA}=20^{\circ}$ LUT using the $22.5^{\circ}$ value and the $45^{\circ}$ LUT using the $42.5^{\circ}$ value.

Supplementary Table 2. Median retrieved surface albedo over ocean and the centre of each $5^{\circ}$ solar zenith angle bin.

\begin{tabular}{|c|c|c|c|c|c|}
\hline & \multicolumn{5}{|c|}{$\mathrm{SZA}\left[{ }^{\circ}\right]$} \\
\hline & 22.5 & 27.5 & 32.5 & 37.5 & 42.5 \\
\hline $\mathrm{O}_{2} \mathrm{~A}$-band & 0.0541 & 0.0357 & 0.0271 & 0.0278 & 0.0230 \\
\hline Weak $\mathrm{CO}_{2}$ band & 0.0421 & 0.0256 & 0.0172 & 0.0181 & 0.0122 \\
\hline Strong $\mathrm{CO}_{2}$ band & 0.0330 & 0.0204 & 0.0139 & 0.0132 & 0.0096 \\
\hline
\end{tabular}

\section{Lookup table input values}

Supplementary Table 3. Input values used for the Nakajima-King tables.

\begin{tabular}{|l|l|l|}
\hline Input Property & Short Name & Values \\
\hline Solar zenith angle & SZA & $20,25,30,35,40,45$ \\
\hline Cloud optical depth, & $\tau$ & $1,2,4,6,8,10,12,14,16,20,35,50$ \\
\hline Droplet effective radius $[\mu \mathrm{m}]$ & $r_{e}$ & $4,6,8,10,12,14,16,20,24,28,32$ \\
\hline Cloud top pressure $[\mathrm{hPa}]$ & $P_{\text {top }}$ & $650,690,730,770,810,850,910,950$ \\
\hline
\end{tabular}

\section{Channel selection algorithm and performance}

We selected a single set of channels for the LUT that would be valid for all meteorological conditions, spacecraft motion, and sounding positions by testing against a sample consisting of every SZA $<45^{\circ}$ MODIS $\tau>1$ footprint from every $50^{\text {th }}$ orbit $(N \sim 230,000)$.

50 The algorithm aimed to select channels whose mean minimised the sample root mean square error (RMSE) against the relevant "truth". For $I_{c, O 2}$ the truth is the L1bSc SoundingMeasurements/rad_continuum_o2, for $I_{c, s t}$ it is SoundingMeasurements/rad_continuum_sco2 and for $I_{a b s, O 2}$ it is the spectrum's $60^{\text {th }}$-ranked superchannel as described in the main manuscript. 
The algorithm first calculates every individual channel's RMSE against the target and then selects the 30 channels with the lowest individual RMSEs. It then calculates the 30-channel mean and returns its RMSE, before removing each channel in turn and calculating the RMSEs of the new 29-channel means. The combination of channels with the lowest RMSE is taken as the 29-channel example, and this process is repeated iteratively down to 3 channels.

The comparison between the targets and the estimates using the selected channels is in Supplementary Figure 2, note that the colours represent logarithmic counts. There is excellent correlation for all three observables, even though each of the LUT

60 values uses the mean of a fixed set of channels whereas the individual channels used in each of the truth values may vary between soundings. In particular, the excellent agreement in Supplementary Figure 2(c) compares with the individual channel spread in the main manuscript Figure 3 shows that the algorithm selected channels with sufficient anti-correlation to reliably reproduce our estimate of $I_{a b s, O 2}$. The median and 5-95\% ranges of the radiance differences are listed in Supplementary Table 4 and the list of channels are in Supplementary Table 5.

65

(a)

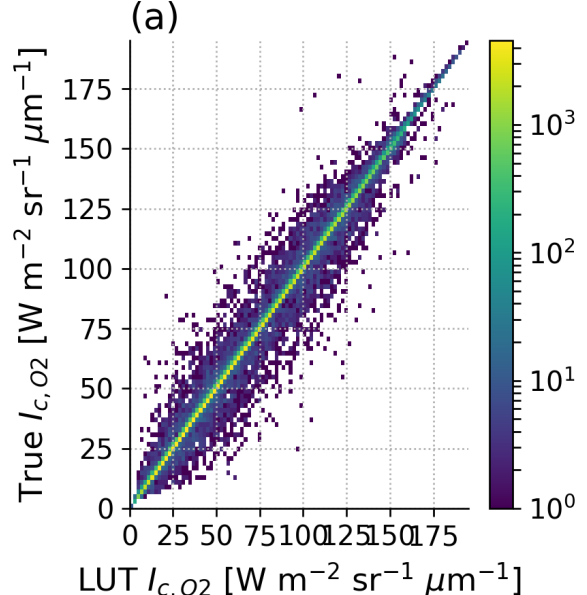

(b)

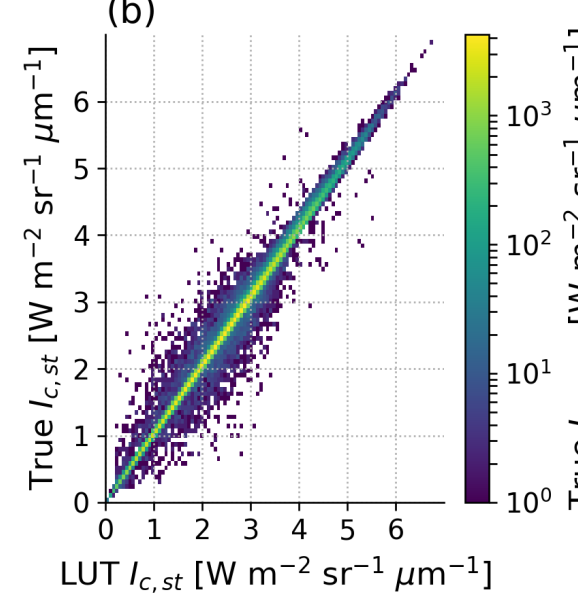

(c)

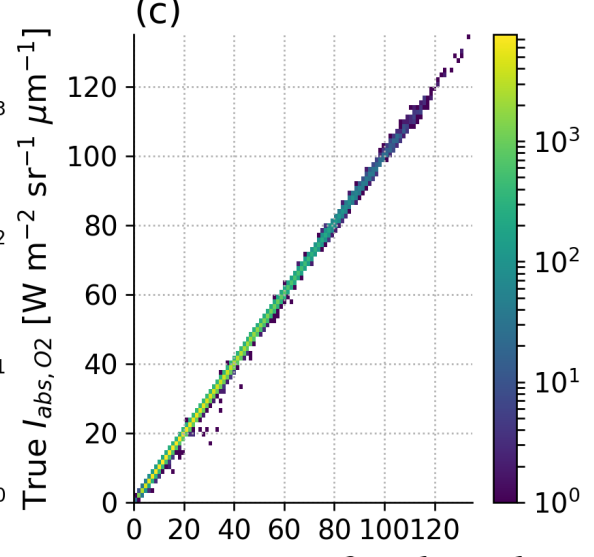

LUT $l_{\mathrm{abs}, O 2}\left[\mathrm{~W} \mathrm{~m}^{-2} \mathrm{sr}^{-1} \mu \mathrm{m}^{-1}\right]$

Supplementary Figure 2. 2d histograms of the target ("true") radiance properties as a function of the means estimated from the fixed channel sets ("LUT"), (a) for the $\mathrm{O}_{2}$ A-band continuum, (b) for the strong $\mathrm{CO}_{2}$ band continuum, (c) for the $60^{\text {th }}$-ranked super-channel, representing an $\mathrm{O}_{2} \mathrm{~A}$-band absorption channel.

70 Supplementary Table 4. Median and 5-95\% range of the differences between the radiance estimates from the mean of the channels used in the lookup table, and the targets.

\begin{tabular}{|l|r|r|r|}
\cline { 2 - 4 } \multicolumn{1}{c|}{} & \multicolumn{4}{c|}{ Percentiles of LUT minus true radiance differences } \\
\hline Radiance property & Median (\%) & 5th percentile (\%) & 95th percentile (\%) \\
\hline$I_{c, O 2}$ & -0.2 & -1.7 & 0.0 \\
\hline$I_{c, s t}$ & -2.3 & -4.7 & -0.5 \\
\hline$I_{a b s, O 2}$ & -0.3 & -2.1 & 1.3 \\
\hline
\end{tabular}


Supplementary Table 5. Channel indices used for each radiance property in the LUT, counting from index 0.

\begin{tabular}{|l|l|}
\hline Radiance property & Channels \\
\hline$I_{c, O 2}$ & $782,827,838,839,889$ \\
\hline$I_{c, s t}$ & $841,842,924,927,937$ \\
\hline$I_{a b s, O 2}$ & $115,113,116,246,329,338,339,380,381,576$ \\
\hline
\end{tabular}

